\title{
EL DEBATE SOBRE LA CONSAGRACIÓN CONSTITUCIONAL DE LA ESTABILIDAD PRESUPUESTARIA EN FRANCIA
}

\author{
JUAN JOSÉ RUIZ RUIZ \\ Profesor Titular de Derecho Constitucional \\ Universidad de Jaén \\ ÁNGEL J. SÁNCHEZ NAVARRO \\ Profesor Titular de Derecho Constitucional \\ Universidad Complutense
}

SUMARIO.
I. Introducción
II. Del principio constitucional a su necesario de- sarrollo técnico
III. El estado de la cuestión, y las inciertas pers- pectivas de futuro

\section{INTRODUCCIÓN}

Probablemente, la única explicación posible sea que «la política es así». Porque cualquier lector de estas crónicas, desde sus inicios en 1998, estará familiarizado con la idea de la «banalización» de la reforma constitucional, expresión formulada por Louis Favoreu en una Francia que, desde 1958, ha modificado su Constitución en 24 ocasiones, quince de ellas entre 1999 y $2008^{1}$. Lo cual marca un acusado contraste con el contexto español, donde se ha generalizado la idea de que la Constitución es prácticamente irreformable ${ }^{2}$.

Y, sin embargo... como examinan otras contribuciones a este número de Teoría y Realidad Constitucional, la iniciativa —impulsada desde el ámbito comunitario, y más

1 Vide TRC, núm. 1 (1998), págs. 205 y ss.; y una última «actualización», en TRC, núm. 22 (2008), pág. 453.

2 Véase, por todos, ESTEBAN, J de y GONZÁLEZ-TREVIJANO, P, que utilizan los términos de «intangibilidad sobrevenida» y la expresión «la reforma como tabú» (en Tratado de Derecho Constitucional, vol. III. Madrid, Servicio de Publicaciones de la Facultad de Derecho de la Universidad Complutense, 2000, págs. 745 y siguientes). 
particularmente por el recuperadísimo eje franco-alemán— de introducir en los textos constitucionales la «regla de oro» del equilibrio presupuestario ha encontrado en España (en las circunstancias de todos conocidas, y que no son objeto de estas páginas) una acogida inmediata, concretada en la reforma constitucional del artículo 135, del 27 de septiembre. Mientras que, en Francia, donde dicho principio se incorporó al artículo 34 de la Constitución con la polémica reforma de 23 de julio de $2008^{3}$, posteriormente no se ha logrado aprobar el desarrollo técnico necesario para asegurar su puesta en práctica. De hecho, el proyecto adoptado por el Gobierno en marzo de 2011 no sólo no ha encontrado todavía el respaldo necesario para incorporarse a la Norma Fundamental, sino que corre un serio riesgo de encallar en los difíciles recodos de la vida política gala, como trataremos de exponer en estas páginas ${ }^{4}$.

\section{DEL PRINCIPIO CONSTITUCIONAL A SU NECESARIO DESARROLLO TÉCNICO}

Porque, en efecto, tras la reforma de 2008, el artículo 34 de la Constitución francesa (que define el ámbito de la ley) atribuyó rango constitucional al objetivo del equilibrio presupuestario. Concretamente, los últimos incisos de dicho artículo señalan, en su redacción vigente, que:

«Las leyes de Presupuestos establecerán los ingresos y los gastos del Estado, en las condiciones y con las reservas establecidas por una ley orgánica.

Las leyes de financiación de la seguridad social determinarán las condiciones generales de su equilibrio financiero y, teniendo en cuenta sus previsiones de ingresos, fijarán sus objetivos de gastos, en las condiciones y con las reservas establecidas por una ley orgánica.

Los objetivos de la acción del Estado se determinarán mediante leyes de programación.

Las orientaciones plurianuales de las finanzas públicas serán definidas por leyes de programación. Se inscribirán dentro del objetivo de equilibrio de las cuentas de las administraciones públicas.

Las disposiciones del presente artículo podrán ser precisadas y completadas por una ley orgánica» ${ }^{5}$.

Así las cosas, la puesta en práctica de este principio exigía articular diversos mecanismos técnicos, que habrían de ser definidos posteriormente. A tal fin, se inició un proceso que desembocaría en el proyecto de reforma constitucional que ahora nos ocupa, y cuya suerte resulta, hoy por hoy, impredecible.

3 Vide la ya citada crónica sobre las circunstancias y el contenido de dicha reforma, en TRC, núm. 22 (2008).

4 Projet de loi constitutionnelle relatif à l'équilibre des finances publiques, adoptado por el Consejo de Ministros el 16 de marzo de 2011.

5 Las cursivas han sido añadidas. 


\section{El PROYECTO DE LEY CONSTITUCIONAL}

\section{A) Contexto y antecedentes: el Informe Camdessus}

Como en otras ocasiones, la puesta en práctica de la mencionada reforma de 2008 provocó, en primer lugar (y, ciertamente, en fuerte contraste con la reforma express del art. 135 de la Constitución española), la celebración de una primera conferencia gubernamental sobre el déficit (enero de 2010), tras la que se formó un grupo de expertos bajo la presidencia de Michel Camdessus, Gobernador honorario del Banco de Francia. La misión inicial de este grupo, en los términos expuestos por el Primer Ministro, era la de formular una serie de propuestas para cumplir el citado mandato constitucional del equilibrio presupuestario de todas las administraciones públicas ${ }^{6}$.

A tal efecto, el Primer Ministro solicitó al grupo que elaborase sus propuestas sobre varias cuestiones:

i) modalidades de funcionamiento de la nueva regla de equilibrio, tratando que las finanzas públicas jueguen un papel de estabilizador automático en caso de crisis y previendo en todo caso las condiciones que permitan volver a una situación de equilibrio a medio término;

ii) los medios de coordinación de las contribuciones de los saldos financieros de las diferentes administraciones públicas a la regla de equilibrio;

iii) el calendario de entrada en vigor de esta regla, teniendo en cuenta el compromiso adquirido por el país para alcanzar el 3\% de déficit público en 2013 y el tiempo preciso para absorber el déficit de manera estructural;

iv) el instrumento jurídico para aplicar la nueva regla a nivel nacional; y

v) la manera más eficaz de asegurar un control de la correcta aplicación de la regla, tanto de carácter preventivo (ex ante) como represivo (ex post).

A pesar de que parte de la doctrina considera ya suficiente la redacción dada en 2008 al art. 34 de la Constitución (en adelante, CF) como inscripción de la estabilidad presupuestaria $^{7}$, una segunda conferencia gubernamental sobre el déficit (el 20 de mayo de 2010) expresó, _ incidiendo en una línea ya planteada por iniciativas parlamentarias desde el año $2001^{8}$ — , la necesidad de proceder a reformar de nuevo la Constitución para elevar al máximo rango la «regla de oro» sobre el déficit público, a la vista de los graves desequilibrios de las finanzas públicas, que tras treinta años de déficit ininterrumpido registraban un déficit público del $7,5 \%$ del PIB y una deuda del $77,6 \%$ del PIB en

6 Vid. lettre du 1er mars 2010.

7 Así, por ejemplo, FELDMAN, J-P Constitutionnaliser l'équilibre budgétaire: nécessité ou artífice? (Institut de recherches économiques et fiscales, 12 May 2011). En contra, algunos autores estiman que la reforma de 2008 se ha revelado insuficiente y que la actual solo trata de dar continuidad a los aspectos esenciales introducidos en 2008, tales como la plurianualidad y la programación. Cfr. CHARPENTIER, J, La limitation des déficits publics dans la Constitution» VIII ${ }^{\text {ème }}$ Congrès National de l'ADFC, Atelier n ${ }^{\circ} 6$, pág. 3.

8 En efecto, la propuesta de incluir una «regla de oro» ya figuraba en las enmiendas Joyandet (sesión del 12 de junio de 2001) al proyecto de ley orgánica reguladora de las Leyes presupuestarias, y también en las propuestas de ley constitucional de Charles de Courson ( $\mathrm{n}^{\circ}$ 609), en sus propuestas de enmienda al proyecto de reforma constitucional de 2008 (sesión de 27 de mayo de 2008), así como en las enmiendas de este parlamentario al proyecto de ley constitucional de 2011 objeto de estas líneas. 
2009 , sin que los momentos de coyuntura favorable se hayan aprovechado para una sustancial reducción del déficit.

De este modo, la inquietud en torno a las graves perturbaciones provocadas por los mercados de deuda y la consiguiente - y preocupante- perspectiva de un aumento del peso de la financiación de la deuda pública por relación al PIB (ya advertida por grupos de trabajo anteriores, como el presidido por Pérebau en 2006), se convierte en el trasfondo dominante del Informe elaborado por la comisión (el Informe Camdessus ${ }^{9}$ ), que se hizo público el 25 de junio de 2010. A pesar de que las Leyes Orgánicas reguladoras de la Ley de Presupuestos (LOLF) y de las leyes de financiación de la Seguridad Social (LOLFSS $)^{10}$ habían supuesto un primer intento por contener y sobre todo racionalizar el déficit, el Informe Camdessus advierte de una serie de deficiencias que aquejan al sistema financiero francés y que es preciso corregir. Entre ellas se señalan:

i) la falta de una regla que asegure la primacía de las leyes plurianuales encaminadas hacia un objetivo de equilibrio de las leyes financieras anuales;

ii) la ausencia de un instrumento que permita someter a las deliberaciones y voto del Parlamento los compromisos europeos;

iii) el alcance limitado del control del Conseil Constitutionnel sobre las leyes financieras $^{11}$;

iv) el arcaísmo de los métodos de ajuste por fluctuaciones coyunturales,

v) la contradicción entre la necesaria disciplina de los procedimientos de adopción de leyes presupuestarias y la dispersión de las fuentes de iniciativa;

vi) la indeterminación de la fecha en la que el objetivo de equilibrio debe ser alcanzado;

vii) la insuficiencia de medios propios para garantizar la sinceridad de las leyes financieras;

viii) la inadaptación de medios de coordinación al servicio de un enfoque global de las finanzas públicas; $y$, finalmente,

ix) la vulnerabilidad de las decisiones fiscales y presupuestarias.

Para ofrecer una respuesta a todas estas necesidades y deficiencias, el Informe elabora una serie de recomendaciones que, en lo que aquí interesa, van desde la propuesta de una reforma constitucional, a la creación de un nuevo tipo de leyes. En concreto, la falta de primacía de las leyes plurianuales sobre las anuales en el cumplimiento de reducción del déficit, la ausencia de un voto del Parlamento sobre los compromisos contraídos con

9 Réaliser l'objectif constitutionnel d'équilibre des finances publiques. Rapport du groupe de travail présidépar Michel Camdessus, Gouverneur Honoraire de la Banque de France, 21 juin 2010. Disponible en los sitios www.gouvernement.fr y en http://www. Ladocumentationfrancaise.fr (apartado de Rapports publics).

10 Loi organique relative aux lois de finances (en adelante, LOLF), de 1 de agosto de 2001; y loi organique relative aux lois de financement de la Sécurité Sociale (en adelante, LOLFSS), de 2 de agosto de 2005.

11 Este es uno de los puntos más debatidos y delicados de la reforma. Parte de la doctrina avisa de la dificultad que entraña garantizar de manera efectiva la eventual constitucionalización de una regla de oro, si no se establecen criterios claramente establecidos a partir de los cuales el Conseil Constitutionnel pueda ejercer su control; pero, sobre todo, la dificultad estriba en que los conceptos y principios financieros están siendo objeto de profundos cambios, y encontrar unos criterios de enjuiciamiento adecuados que sirvan como canon de constitucionalidad es una tarea que queda por hacer. Vid. BOUVIER, M, «La règle d'or: un concept à construire?», Revue Française de Finances Publiques, núm. 113, Février 2011, pág. V. 
la UE y el insuficiente alcance del control del Conseil constitutionnel sobre las leyes financieras, llevan a formular una nueva redacción de los arts. 34 CF y 47-1 CF.

Así, la nueva redacción propuesta para el art. 34 contempla reemplazar las «leyes de programación» de las finanzas públicas (LPFP), previstas en 2008, por un nuevo tipo de ley, dentro del sistema de fuentes que, con el carácter de ley orgánica, recibe el nombre de «ley marco de programación de las finanzas públicas» $\left(\mathrm{LCPFP}^{12}\right)$, cuya función estribaría en «determinar las normas de evolución y las orientaciones plurianuales de las finanzas públicas con vistas a asegurar el equilibrio de las cuentas de las administraciones públicas», y cuyo rasgo específico consistiría en que sus disposiciones se imponen, ope Constitutione, a las leyes presupuestarias y de financiación de la Seguridad social.

Esta primacía de la ley marco de programación sobre las leyes presupuestarias y de financiación de la Seguridad social vendría a ser la principal novedad que se pretende introducir respecto a las actuales leyes (también plurianuales) de programación de las finanzas públicas, de las que se ha hecho uso ya en dos ocasiones ${ }^{13}$. En ambos casos, el carácter plurianual pone fin al sistema anterior que fijaba anualmente la programación de las finanzas públicas en el programa de estabilidad que se envía a la Comisión Europea y en el informe económico, social y financiero que, como anexo, incluye el proyecto de ley de presupuestos. Con el nuevo sistema, la programación es debatida y votada en el Parlamento sin necesidad de que sea revisada cada año. La otra nota esencial de las leyes de programación creadas en 2008 es su carácter de ley horizontal, en el sentido de que en ella se comprenden no solo las cuentas del Estado, sino también las de las administraciones de la seguridad social y las colectividades territoriales, así como la financiación del seguro de desempleo y los regímenes complementarios de jubilación ${ }^{14}$.

Por otra parte, el rango jerárquico de esta nueva categoría de leyes sería inferior al de las leyes orgánicas, y superior en algunos de sus contenidos al de las leyes ordinarias de presupuestos y de financiación de la Seguridad Social ${ }^{15}$. La inferioridad de rango respecto a la ley orgánica resulta de lo dispuesto en el proyecto de ley constitucional, ya que tanto el contenido como la periodicidad de las leyes-marco de programación vendrán determinados por una ley orgánica (propuesta ya contenida en el Informe ${ }^{16}$ y en el art. 4 del

12 Loi-cadre de programmation pluriannuelle des finances publiques (LCPFP).

13 La última (ley de 28 de diciembre de 2010 de programación de las finanzas públicas para el período 2011-2014) proponía una estrategia para alcanzar el doble objetivo de reducir el déficit al 6\% del PIB en 2011 y alcanzar el $3 \%$ en 2013, de acuerdo con los compromisos contraídos con la UE por el pacto de estabilidad.

14 Esta inclusión de la Seguridad social y las colectividades territoriales en el equilibrio presupuestario diferencia la regla del art. 34 CF de la que se ha introducido en Alemania en la reforma de agosto de 2009, que solo afecta al Bund y Länder pero no a los organismos de la Seguridad social ni a los ayuntamientos, cuya capacidad de endeudamiento es, por lo demás, muy débil.

15 El mayor rango jerárquico de las leyes-marco de programación respecto de las leyes de presupuestos y ordinarias y el menor rango respecto de la ley orgánica que establece su ámbito material, modificaría la pirámide de fuentes existentes hasta ahora, como observa Le Pourhiet: «La Constitución fija simplemente un objetivo de equilibrio; una ley orgánica, que debe ser conforme a la Constitución, determina seguidamente las modalidades de elaboración de las leyes-marco de equilibrio de los presupuestos; éstos deben a su vez respetar la Constitución, las leyes orgánicas y las leyes-marco de equilibrio de los presupuestos, cada escalón haciendo intervenir al Conseil Constituionnel» (LE POURHIET, A-M, «L'équilibre des finances publiques dans la Constitution», Notes et Etudes de la Fondation Res Publica, 4 Avril 2011).

16 El cual proponía una nueva redacción del art. 47-1 CF: «El Parlamento vota los proyectos de ley-marco de programación de los presupuestos generales en las condiciones previstas por una ley orgánica». 
proyecto de reforma). No obstante, su procedimiento de aprobación es idéntico al de las leyes ordinarias de presupuestos ${ }^{17}$; de hecho, el proyecto de reforma dispone que los proyectos de ley-marco de programación gozarán de prioridad en su tramitación al igual que los proyectos de ley de presupuestos, modificando de este modo la redacción actual del art. 39 CF. Igualmente el Gobierno gozaría en la tramitación de estas leyes del procedimiento-sanción de inacción de las cámaras previsto en el art. 47 CF (que impone plazos a ambas cámaras y permite recurrir a una comisión mixta con una sola lectura del proyecto).

Ahora bien, desde el punto de vista material, la particularidad de la nueva categoría de las leyes-marco de programación es que aquellas disposiciones que especifique la ley orgánica reguladora de este tipo de fuente se impondrán a las leyes de presupuestos y a las leyes de financiación de la Seguridad Social (SS). Es decir: la validez de las leyes de presupuestos y de financiación de la SS no está condicionada por todo su contenido, sino solo por aquellas partes o aspectos materiales definidos por la ley orgánica, a los que se reconoce pues un rango superior, tal y como expresa el propio proyecto de ley de reforma ${ }^{18}$.

A partir de este nuevo tipo normativo (la LCPFP), el control del respeto a sus disposiciones correspondería al Conseil Constitutionnel, que examinaría su conformidad con el principio constitucional de equilibrio presupuestario de las administraciones públicas contenido en el art. $34 \mathrm{CF}$ tras la reforma de 2008 y, eventualmente, examinaría la trayectoria hacia el objetivo de equilibrio fijado por el legislador. Ello exigiría no obstante -apunta el Informe-, incluir este nuevo tipo normativo entre los que se someten "de oficio» al control (previo) de constitucionalidad, incluyendo a tal efecto un nuevo inciso al final del primer párrafo del art. $61 \mathrm{CF}$.

Además de esta modalidad de control, el Conseil podría ser instado a pronunciarse sobre la conformidad de las leyes presupuestarias (LF) y de financiación de la Seguridad social (LFSS) a la jerarquía de normas una vez ya aprobadas (art. 61, segundo párrafo, CF). De este modo el Consejo garantizaría que dichas leyes respetan el techo de gasto y el umbral mínimo de ingresos impuestos por la LCPFP en vigor, y su decisión tendría, en su caso, los efectos usuales de una declaración de inconstitucionalidad global, por vulnerar el mandato constitucional de equilibrio de la ley de presupuestos. Asimismo, el Informe proponía un control sobre las desviaciones del objetivo marcado por la LCPFP consistente en que, mientras dure la ejecución de las leyes de presupuestos o de las LFSS, todo desfase de gastos y/o de ingresos respecto a la trayectoria prevista en la LCPFP debería ser corregido y compensado, bien antes de la finalización del ejercicio (si los desvíos se han comprobado durante su ejecución) o bien durante los dos ejercicios siguientes (si se ha comprobado al final de la ejecución). Esta propuesta se inspira en la cuenta nocional alemana de control de desviaciones, consistente en una cuenta de amortización en la que se incluye el desfasamiento respecto a la trayectoria prevista. En el caso alemán, si el saldo de esta cuenta nocional sobrepasa el $1 \%$ del PIB se deben adoptar medidas correctoras de manera inmediata (salvo en caso de recesión) para conciliar el desfase acumulado en los

17 Vid. LE POURHIET, A-M, «'équilibre...», op. cit., s.p.

18 Assemblée Nationale, $\mathrm{n}^{\circ} 3253$, Projet de loi constitutionnelle relatif à l'équilibre des finances publiques, pág. 6. 
fondos, de manera adicional al control presupuestario que corresponda al año en cuestión $^{19}$.

\section{B) Contenido: del Informe al proyecto de reforma}

Frente al —al menos, cuantitativamente- limitado alcance de la reforma constitucional operada en España, y ceñida al artículo 135, la complejidad del proyecto de reforma constitucional aprobado, hasta el momento, por las dos Cámaras del Parlamento francés se hace patente al considerar que afecta, ni más ni menos, a doce artículos de la Constitución de $1958^{20}$. De cualquier modo, sus principales innovaciones pueden agruparse en unos pocos puntos, que pasamos a analizar.

\section{a) Una nueva categoría de Ley: la loi-cadre de programmation des finances publiques} (LCPFP)

Como se ha apuntado en el apartado anterior, el Informe Camdessus suscitó el debate político y doctrinal sobre una propuesta que viene a modificar el sistema de fuentes vigente en el ordenamiento francés. Se trataba, concretamente, de introducir una nueva categoría legislativa (las leyes marco de programación de finanzas públicas), como instrumento de control del déficit. Un tipo de ley, de un lado, jerárquicamente superior a las leyes presupuestarias y de financiación de la Seguridad social (LFSS) ${ }^{21}$, y, de otro, de naturaleza plurianual.

En virtud de esa doble naturaleza la ley marco de programación aparecía en el Informe como una ley de carácter plurianual en la que se fijan (en euros constantes para cada año de programación):

i) por un lado, los techos del nivel de gasto del Estado que recae, respectivamente, en los ámbitos de la ley presupuestaria y de la LFSS;

ii) por otro, el umbral mínimo de ingresos del Estado y de la Seguridad social, atendiendo a factores distintos a la evolución espontánea tales como las medidas fiscales, a fin de evaluar las vías y medios incluidos en la ley presupuestaria.

La Exposición de motivos del proyecto de ley constitucional, aprobado el 16 de marzo de 2011, recoge esas mismas naturaleza y funciones, sin ninguna variación respecto al Informe; únicamente viene a concretar dos extremos relativos a la programación que éste había dejado abiertos. En primer lugar, dicha Exposición de motivos propone que la duración mínima de una ley-marco de programación sea de 3 años (formulando el desiderátum de que sería más funcional una duración de cinco); sin embargo, la redacción del art. 34 CF propuesta por el proyecto deja finalmente la precisión de este extremo a la ley orgá-

19 Vid. Deficit Rule Reform Team, Federal Ministry of Finance-Economics Department, Reforming the Constitutional Budget Rules in Germany, September 2009, paper.

20 Concretamente, a los artículos 34, 39, 42, 46-1, 47, 47-1, 48, 49, 61, 70, 72-2 y 88-8 (algunos de los cuales se introducen en el texto vigente).

21 Este último tipo de leyes fue introducido con la reforma constitucional de 1996 y ya suscitó reparos su originaria denominación, que revelaba claramente el objetivo de equilibrar los presupuestos de la Seguridad Social. 
nica reguladora del nuevo tipo de fuente, renunciando por tanto a introducirla en la Constitución ${ }^{22}$. En segundo lugar, la misma Exposición precisa y aclara que el legislador sólo estará vinculado jurídicamente a los dos tipos de disposiciones que integran necesariamente el contenido material de una ley-marco de programación (techos de gasto y umbral mínimo de ingresos): todas las demás disposiciones de una ley de este tipo no vinculan jurídicamente, sino que constituirán disposiciones meramente programáticas que, por tanto, sólo vinculan políticamente al legislador.

Según el Informe, la primacía de la ley-marco de programación basada en la superior jerarquía que le reconocería la Constitución aproximaría estas leyes a las leyes orgánicas, e impediría al legislador exceder el techo de gastos o disminuir el nivel de ingresos previstos por la LCPFP, salvo que aumenten los ingresos o disminuya el gasto en la misma proporción. En caso de degradación de la coyuntura este mecanismo permitiría, según el grupo de expertos, disponer de todos los márgenes de maniobra presupuestarios para dejar actuar los estabilizadores automáticos. El proyecto de ley constitucional asume íntegramente esta concepción al disponer, por un lado, que no podrá adoptarse definitivamente una ley de presupuestos ni de financiación de la Seguridad Social en ausencia de ley-marco de equilibrio presupuestario aplicable al ejercicio en curso (dando nueva redacción a los artículos 47 y 47-1 CF); mientras que, por otro, reconoce el superior rango de algunas de sus disposiciones (las que determine una ley orgánica reguladora del tipo legal) al incluir a esta nueva categoría de leyes entre las sometidas al control de oficio del Conseil Constitutionnel, el cual no sólo examinará la conformidad de estas leyes con la Constitución, sino también con la propia ley orgánica configuradora de su contenido.

Por otra parte, el Informe Camdessus contemplaba asimismo que la ley marco de programación tuviera un contenido adicional a la parte dispositiva, en forma de informe anexo que debería detallar el esfuerzo estructural y la trayectoria del saldo estructural y la del saldo efectivo. Puesto que estas especificaciones son las que deben incluirse en el programa de estabilidad que debe enviarse a la Comisión Europea y al Consejo, el Parlamento se pronunciaría, votando la ley-marco, sobre el plan de estabilidad ${ }^{23}$.

En este sentido el Informe Camdessus proponía que el informe anexo expusiera además los grandes ejes de la política fiscal, así como las reformas estructurales en marcha para el período de aplicación de la ley-marco de programación. No obstante, el proyecto de revisión constitucional ha considerado que el debate y votación del proyecto de

22 La Asamblea Nacional es la que, al final, incluye en el artículo 34 CF un período mínimo de duración de 3 años (véase el Projet de Loi constitutionnelle adopté par l'Assemblée Nationale, rélatif à l'équilibre des finances publiques, transmis par M. le Premier Ministre à M. le Président du Sénat, pág. 3).

23 En todo caso, la votación de la ley-marco solo afectaría a su parte dispositiva, ya que -si bien el informe incluiría aspectos como la trayectoria de las cuentas del conjunto de las administraciones públicas, comprendiendo pues la evolución de los saldos públicos, de la deuda pública y de los gastos e ingresos fiscales- quedan fuera de la competencia del legislador las cuentas de las colectividades territoriales y de las administraciones de la seguridad social que recaen fuera del ámbito de las leyes de financiación de la Seguridad social.

Sin embargo el contenido de este anexo-informe de la ley marco abarcaría otros aspectos que exceden desde luego el plan de estabilidad, como la norma de evolución de gastos del Estado, el Objetivo nacional de gastos de seguro médico, la norma de evolución de los concursos a las colectividades territoriales y el reparto de techos de gasto por partidas del presupuesto general del Estado, actualmente recogidos en la ley de programación de 9 de febrero de 2009 (véase el reiterado Informe Camdessus, pág. 13). 
programa de estabilidad tiene entidad propia ${ }^{24}$ y que no debe anexarse como informe al proyecto de una ley-marco de programación, sino que debe enviarse a las dos cámaras del Parlamento antes de su envío a la Comisión Europea en virtud de una nueva obligación, impuesta al Gobierno mediante la introducción de un nuevo art. 88-8 en la Constitución.

Resulta indudable que la ventaja de una ley-marco de programación es la vinculación del legislador ordinario presupuestario a los objetivos y límites establecidos en ella, así como la estabilidad que representa para el período afectado. Además, al tratarse de límites de mínimos, nada impide que el legislador ordinario acelere en un año determinado la consecución o la progresión del equilibrio financiero, por ejemplo con motivo de la formación de un nuevo gobierno. Sin embargo esta flexibilidad presupuestaria no existe respecto a los límites de mínimos, sustrayendo a Gobierno y Parlamento unas posibilidades de las que hasta ahora han venido gozando, salvo un cambio de mayoría parlamentaria o un acontecimiento internacional que obligue a revisarla. Ello implica un elemento de rigidez que puede condicionar la puesta en marcha de determinadas políticas, y que el Informe considera el precio a pagar a cambio de alcanzar el equilibrio estructural deseable, con el fin de evitar sacrificar la independencia frente a los mercados de deuda.

b) El monopolio de las leyes presupuestarias para establecer medidas fiscales y tributarias

La segunda gran novedad del proyecto de reforma constitucional propuesto por el Gobierno, siguiendo igualmente el Informe del grupo Camdessus, afecta también al sistema de fuentes del Derecho. Concretamente, trata de acabar con la dispersión de fuentes normativas que pueden introducir medidas fiscales o que afecten a los ingresos de la Seguridad social, instaurando en la Constitución una regla de jerarquización que consagraría un auténtico monopolio de las leyes presupuestarias (general y de la Seguridad Social) en materia fiscal.

Según el Informe, esa regla constituiría un criterio racionalizador que impediría a la legislación sectorial establecer disposiciones contradictorias o mutuamente competitivas. En consecuencia, se suprimiría la mención a «la base imponible, las tasas y las modalidades de recaudación de impuestos de cualquier naturaleza» que el artículo 34 hace en su párrafo quinto actual, introduciendo un nuevo párrafo según el cual «en las condiciones y sin perjuicio de lo que disponga una ley orgánica, las leyes de presupuestos y las leyes de financiación de la Seguridad social fijan las reglas relativas a la base imponible, las tasas y las modalidades recaudatorias de impuestos de cualquier naturaleza».

Esta modificación implicaría incluir en el ámbito material de la ley de presupuestos las cotizaciones a la Seguridad social, que hasta ahora han venido siendo reguladas por normas reglamentarias, lo cual —como advierte el propio Informe Camdessus — alteraría la

24 En efecto, como recuerda Servière, programa de estabilidad y equilibrio estructural representan cosas distintas: la denominada «regla de oro» del art. 34 CF está dirigida a alcanzar el equilibrio presupuestario, mientras que el programa de estabilidad es una regla de déficit público autorizado que conforme los criterios de Maastricht limita el déficit al 3\% del PIB y el endeudamiento al 60\% del PIB. Vid. Samuel-Fréderic Servière, Réforme constitutionnelle d'équilibre des finances publiques. La rigueur en trompe l'oeil?, Fondation iFrap, 12 mai 2011. 
línea divisoria entre poder reglamentario y poder legislativo, ya que el Parlamento pasaría a ocuparse de la aprobación de esas exacciones que vienen a engrosar otras que ya vienen siendo fijadas mediante ley, como por ejemplo la tasa de los elementos que integran la contribución social generalizada o la tasa de contribución para la devolución de la deuda social (CRDS).

Por todo ello, este punto ha constituido el mayor motivo de fricción entre el Gobierno y las mayorías que lo apoyaban en ambas Cámaras, y finalmente ha sido eliminado del texto. Se alegaron para ello razones de tanto peso como que ello laminaría no sólo el derecho de iniciativa de los parlamentarios (en tanto la iniciativa de las leyes presupuestarias se reserva al Gobierno), sino también la competencia del legislador ordinario, que quedaría imposibilitado de incluir medidas de política fiscal en leyes dedicadas, por ejemplo, a regular una materia o a incentivar un determinado sector de actividad ${ }^{25}$. Unas críticas que también llegaron desde el ámbito académico ${ }^{26}$.

c) Mecanismos de evaluación y de alerta temprana y competencia del Tribunal de Cuentas

En otro orden de cosas, y a fin especialmente de reforzar la credibilidad y sobre todo la fiabilidad de los costes asociados a nuevas disposiciones legislativas y de entablar un diálogo contradictorio con el Gobierno, el Parlamento según numerosas voces debería de estar dotado en materia de evaluación de expertos propios o bien debería de dirigirse a instancias de evaluación externas que auditen las cuentas del Estado ${ }^{27}$. No sólo sería conveniente una evaluación ex ante sino también ex post que permita revisar el impacto efectivo de medidas fiscales y de ingresos de Seguridad social.

Precisamente, en la línea de reforzar el control ex ante del cumplimiento de los objetivos establecidos en la ley-marco de programación se recogen una serie de mecanismos que el Informe Camdessus designa genéricamente como de «alerta temprana» ante el Parlamento. Dichos mecanismos de alerta varían según se trate de examinar la ejecución del presupuesto estatal, del presupuesto de la Seguridad social o de la ejecución presupuestaria de las colectividades territoriales.

En el caso de la ejecución presupuestaria de los órganos estatales, el Tribunal de Cuentas ya tiene la obligación de elaborar anualmente un informe sobre la ejecución del

25 En efecto esta medida fue, inicialmente, descartada por la Commission des lois de la Asamblea Nacional, a propuesta del ponente; luego, recuperada en el texto votado por el Pleno de la Cámara, en primera lectura; posteriormente, rechazado por la mayoría del Senado; y, finalmente, eliminado del texto aprobado por ambas Cámaras. Véanse los documentos correspondientes: projet de loi; Rapport elaborado para la Comisión de leyes de la Asamblea por el diputado Jean-Luc Warsmann (13 de abril de 2011); el texto adoptado por la Asamblea en primera lectura (10 de mayo), el Avis du Sénat (n 595, de 9 de junio 2011); o el texto votado, en términos idénticos, por ambas Cámaras (13 de julio de 2011; todos ellos, disponibles en www.legifrance.gouv.fr).

26 Así, en su audición ante la Asamblea Nacional, Guy Carcassonne consideraba que «este monopolio es problemático [...] ¿Cómo puede hablarse seriamente de política de vivienda, educativa o sanitaria sin plantear en ningún momento la cuestión de los medios? (...) Ello supondría una reforma implícita del artículo 20 de la Constitución, que debería establecer: «El Ministro de Hacienda determinará la política de la Nación» (citado en un artículo de Les Echos «Les députés opposés au monopole des lois de Finances sur les mesures fiscales», n 20911 du 13 Avril 2011, pág. 3).

27 Vid. SERVIÈRE, S-F, Réforme constitutionnelle d'équilibre..., op. cit., s.p. 
año anterior en virtud del art. 58-4 de la Ley Orgánica reguladora de la Ley de Presupuestos (LOLF). Su presentación en el Parlamento podría servir para tener un debate sobre la ejecución presupuestaria de los primeros meses del ejercicio en curso y podría ser la ocasión para analizar las tendencias que se dibujan en el horizonte. El papel del Tribunal de Cuentas sería el de colaborar en la prevención y de alertar de posibles desvíos. En el caso de la Seguridad Social, el debate presupuestario de primavera en el que las cámaras examinarían la Ley marco de programación presupuestaria (LCPFP) podría servir para incluir la información proporcionada por la comisión de cuentas de la Seguridad Social que, más allá de manifestarse sobre los resultados del año anterior, podría informar sobre los primeros resultados del año en curso. En cuanto a las colectividades territoriales, el debate parlamentario de primavera no puede suministrar información suficiente sobre los primeros meses del año, aunque el Informe proponía que el Tribunal de Cuentas se dote de medios para valorar los primeros datos.

En cualquier caso, para el Informe Camdessus el debate de «alerta temprana» sobre la ejecución presupuestaria debería servir para elaborar conclusiones que, acompañadas de las recomendaciones pertinentes sobre el grado de cumplimiento de la programación plurianual de la LCPFP, darían cierta continuidad al Informe preliminar al debate de orientación presupuestaria que exige el artículo 58-3 de la LOLF.

De cualquier modo, estas propuestas sobre un control en los primeros meses de ejecución presupuestaria por parte del Tribunal de Cuentas no fueron acogidas por el proyecto inicial presentado por el Presidente de la República. A pesar de lo cual, la Asamblea Nacional en primera lectura introdujo una reforma del art. 47-2 CF según la cual «el Tribunal de Cuentas asiste al Parlamento y al Gobierno en la aplicación de las leyesmarco de equilibrio de los presupuestos...».

Lo que sí ha previsto el proyecto de reforma constitucional (art. 12, que introduciría un nuevo art. 88-8 CF) ha sido la celebración en el Parlamento, como ya se ha dicho, de un debate sobre el programa de estabilidad, que se sustanciaría a través del debate sobre declaraciones del gobierno, en el que cabe el voto de mociones o resoluciones. Según el nuevo artículo, los proyectos del programa de estabilidad se transmitirán al Parlamento en torno al 15 de abril y se debatirán con la posibilidad de que las Cámaras voten resoluciones sobre los mismos, de acuerdo con el art. 34-1 CF, salvaguardando la facultad del Gobierno de efectuar una declaración seguida o no de un voto en los términos del art. 50$1 \mathrm{CF}$, que en todo caso no compromete la responsabilidad política gubernamental. Esta previsión no constituye empero novedad alguna, puesto que la ley de programación para el período 2011-2014 ya dispone en su art. 14 que «a partir de 2011, el Gobierno dirige al Parlamento, al menos dos semanas antes de su transmisión a la Comisión europea (...) el proyecto de programa de estabilidad. El Parlamento debate este proyecto y se pronuncia mediante un voto». Lo que pretende por tanto el proyecto de reforma es constitucionalizar el debate sobre el programa de estabilidad, actualmente previsto a nivel infraconstitucional. Ahora bien, lo que no contempla el art. 12 del proyecto es que el programa de estabilidad se deba votar obligatoriamente en las cámaras, ya que solo se limita a exigir un debate seguido de eventuales mociones o resoluciones votadas por los grupos parlamentarios. En este sentido, el Informe de la Comisión de presupuestos del Senado sobre el programa de estabilidad de 2011-2014, advertía de que la ausencia, en el texto del proyecto de reforma constitucional, de la exigencia de su votación en las cá- 
maras debía ser corregida argumentando que «desde el punto de vista de la soberanía nacional, es indispensable que el documento que compromete la estrategia de los presupuestos sea no solamente examinado y debatido en el Parlamento antes de su transmisión a las instituciones de la Unión Europea, sino que igualmente sea objeto de una votación» ${ }^{28}$.

d) La fecha de retorno al equilibrio presupuestario

El Informe Camdessus consideraba imprescindible la fijación de una fecha en la que obligatoriamente se debe alcanzar el objetivo de equilibrio presupuestario; y ello, tanto desde el punto de vista de la credibilidad ante la opinión pública como desde el punto de vista del juicio de constitucionalidad de la regla de equilibrio impuesto por el art. $34 \mathrm{CF}$.

El Informe se decantaba de este modo por una solución ya adoptada en Alemania en agosto de $2009^{29}$, donde el gobierno federal se obliga a no superar un $0,35 \%$ del PIB en 2016 (y los Länder en 2020). La regla alemana presenta, según el Informe, el mérito de que, al definirse en términos estructurales, además de evitar que las fluctuaciones coyunturales puedan poner en riesgo el objetivo del equilibrio, posibilita que en fases bajas del ciclo entren en juego estabilizadores automáticos y que en fases de crecimiento y expansión no se produzcan desvíos del objetivo del déficit.

Aun reconociendo el valor que tiene fijar una fecha, el grupo de expertos se pronunciaba por dejar a la discrecionalidad del legislador la inclusión en la ley marco (en el informe-anexo) de la fecha en la que se deba alcanzar el objetivo de equilibrio en las cuentas de las administraciones públicas establecido en el art. $34 \mathrm{CF}^{30}$, dejando el interrogante de si debía igualmente figurar en la ley orgánica reguladora de las leyes-marco. En cualquier caso se decantaba de modo claro por establecer la fecha de equilibrio.

El texto del proyecto de ley constitucional de revisión guarda por el contrario un significativo silencio sobre este punto, ya que no menciona la fijación de una fecha ni en la Constitución, ni en la ley orgánica reguladora de las leyes-marco, ni tampoco en estas últimas.

\section{LAS DIFICULTADES: EL DEBATE POLÍTICO Y PARLAMENTARIO}

En todo caso, ni la general constatación de la profundidad de la crisis económica -reflejada, incluso, en el ámbito comunitario con decisiones en las que se aprecia un destacado liderazgo francés y, sobre todo, alemán- ni la existencia de un Informe previo elaborado por un grupo de expertos han podido asegurar la existencia de un consenso político en torno a las medidas propuestas por el proyecto de reforma constitucional que nos ocupa. En efecto, desde el principio - y como ocurriera ya en la reforma de 2008-

28 Vid. MARINI, P., Rapport d'information n 456 fait au nom de la commission des finances sur le projet de programme de stabilité transmis par le Gouvernement à la Commission européenne conformément à l'article 121 du traité sur le fonctionnement de l'Union européenne, 26 avril 2011, pág. 9.

29 Fueron objeto de reforma los arts. 109, 109a, 115 y 143d de la Ley Fundamental.

30 MARINI, P., ob. cit. pág. 13. 
las formaciones políticas han sostenido posiciones diferentes e, incluso, enfrentadas. Algo aún más explicable a la vista de que este año 2012 es un «año electoral», en el que han de celebrarse unas elecciones presidenciales que a priori parecen presentarse muy reñidas, y que luego — como es habitual desde la reforma que estableció el mandato presidencial de cinco años- condicionarán en altísimo grado las inmediatas elecciones legislativas.

De este modo, la opinión pública gala ha presenciado un intenso debate político, con argumentos enfrentados, y que ha sido determinante para explicar la situación actual del proyecto, como expondremos brevemente a continuación.

\section{A) El debate político}

Es preciso comenzar este epígrafe poniendo de manifiesto una circunstancia que explica la íntima conexión entre el debate político y las dificultades parlamentarias en esta materia. Porque el debate político no se centra, como ha ocurrido en algunos momentos en España, en la existencia de una crisis cuya gravedad, como ya se ha apuntado, es comúnmente asumida. En gran parte, la discrepancia central radica en que la mayoría considera que la dimensión de la crisis requiere una respuesta al máximo nivel institucional (o, dicho de otro modo, una reforma constitucional que lleve a la Norma Fundamental los criterios y mecanismos necesarios para asegurar la estabilidad de las cuentas públicas); mientras que la oposición estima que, por el contrario, tal instrumento es una «excusa» o una «política de comunicación». Desde este último punto de vista, la estabilidad presupuestaria es una cuestión de voluntad política: quienes hacen y gestionan los presupuestos tienen en su mano la posibilidad de asegurar dicha estabilidad, y si ello no se ha hecho así —en particular, si la mayoría que ha gobernado Francia en las últimas décadas no lo ha hecho así-, no cabe ahora que busque un refugio constitucional para los riesgos que ha asumido políticamente. Al margen, naturalmente, de otros argumentos relativos a los concretos mecanismos que se pretende instaurar o a las concepciones globales que subyacen a la iniciativa.

Tales argumentos se han repetido machaconamente, tanto en la opinión pública como en sede parlamentaria, y tanto en un campo como en otro. De esta forma, en cuanto a la necesidad o conveniencia, de parte de la oposición se ha dicho que «las disposiciones presentadas son, en primer lugar, inútiles. No es necesario modificar la Constitución para asegurar la consolidación de las cuentas públicas: esto es, ante todo, una cuestión de voluntad política. Existe cierta hipocresía cuando se propone un texto pretendidamente riguroso y, en la práctica, se es incapaz de asegurar el respeto a las reglas que ya existen, como el criterio europeo del $3 \%$ del PIB, y cuando se apresuran a asegurar que las leyes-marco [plurianuales] serán revisables anualmente»; es más: «es hipócrita incluso en el método. Se presenta como de extrema urgencia, cuando no se aplicará sino a partir de 2013», lo que — dicho sea de paso— supone posponer «la resolución del problema a la próxima legislatura y, además, tratar de imponer reglas al próximo Gobierno» ${ }^{31}$. En

31 Intervención del Diputado Olivier Dussopt (Grupo SRCDG: Socialista, Radical, Ciudadano e independientes de izquierda), en la reunión de la Comisión de leyes de la Asamblea Nacional, el 13 de abril de 2011. Vide el Rapport citado, pág. 39. 
la misma línea, por ejemplo, el Senador socialista Bernard Frimat afirmaba que «la inscripción en la Constitución no es una solución. Hace falta una voluntad política»; y el proyecto en discusión es «una operación de comunicación [política]», y la mayoría está «en la peor posición para dar lecciones de virtud presupuestaria» ${ }^{32}$.

Desde otros puntos de vista, se subrayaba que la aprobación de la reforma supondría dotar al Consejo Constitucional de «una nueva función, la de verificar que las leyes presupuestarias son conformes a las leyes-marco. Cabría preguntarse sobre qué criterios habría de fundarse, tratándose de la traducción financiera de orientaciones eminentemente políticas; de otra parte, tampoco es clara la credibilidad y la legitimidad del Consejo para pronunciarse sobre tales asuntos, a menos que se reforme totalmente el procedimiento de designación de sus miembros». Pero, en definitiva, la cuestión de fondo es la misma por lo que, volviendo al argumento anterior, «es una mala respuesta a una cuestión real, como es la de la necesaria reducción de los déficits públicos. La solución no es una transferencia del poder de decisión del ámbito político al jurídico, sino por el contrario una firme voluntad política de reducir los déficits, que la actual mayoría no ha demostrado hasta ahora» ${ }^{33}$.

Los mismos argumentos aparecían, si bien en un marco aún más global, cuando un Senador ecologista afirmaba que «estamos particularmente alarmados al ver que, considerándose a sí mismo incapaz de resistir a los clientelismos fiscales, el Gobierno prefiere hacer dejación de sus responsabilidades y someterse enteramente a la ilusoria protección de la Constitución», en un «dramático debilitamiento de la política», porque «equilibrar las cuentas públicas es una necesidad... [pero] no la única. También existen urgencias medioambientales y sociales», por lo que «no saldremos de esta crisis sistémica sin un proyecto de sociedad global, y no solamente financiero» ${ }^{34}$.

$\mathrm{Ni}$ que decir tiene que, desde la mayoría, tales argumentos se rechazan subrayando la gravedad de la crisis, la necesidad de respuestas contundentes y apelando a la responsabilidad de todos (denunciando, de paso, la de quienes se oponen). Así, el ponente en la Comisión legislativa de la Asamblea afirmaba que «no se puede vivir a crédito eternamente», pero que «no se trata de imponer las orientaciones políticas de un momento; al contrario... A la mayoría que salga de las urnas en 2012 corresponderá la tarea de definir una trayectoria para las finanzas públicas, en una ley-marco que votará para, al menos, tres años»; mientras que, de otra parte, «el Consejo Constitucional no tendrá dificultades para ejercer su control. No se tratará de un control de oportunidad, de ingresos o de gastos, sino de un control de conformidad de la[s] leyes presupuestarias... con la ley-marco, asegurando que la mayoría respeta las reglas que se haya autoimpuesto» ${ }^{35}$.

En todo caso, y como tantas otras veces hemos observado en muchas otras crónicas, los debates no modificaron las posiciones enfrentadas de ambos polos políticos, como ponen de manifiesto las votaciones parlamentarias. Ello explica, como veremos inmediata-

32 Sénat, Compte rendu analytique officiel du 11 juillet 2011 (explicaciones de voto antes de la votación final global sobre el texto, en $2^{a}$ lectura).

33 Diputado René Dosière, del mismo Grupo Parlamentario, en la misma sesión de la Comisión legislativa de la Asamblea (loc. cit., págs. 40-41).

34 Jean Desessard, en la $1^{\mathrm{a}}$ lectura del Senado (Compte rendu analytique officiel du 15 juin 2011).

35 J.-L. Warsmann, en el citado debate en la Comisión de leyes de la Asamblea Nacional (13 de abril de 2011; Rapport, pág. 41). 
mente, la difícil tramitación del proyecto hasta la fecha... y las dificultades que, aún hoy, hacen que su destino final parezca más que incierto.

\section{B) Las dificultades en su tramitación}

Porque, en efecto, las posiciones —inamovibles, casi unánimes e irreductiblemente enfrentadas - de mayoría y oposición se han reflejado en las sucesivas votaciones parlamentarias realizadas hasta este momento, y que incluyen tres lecturas del texto en la Asamblea Nacional y dos por parte del Senado.

De cualquier modo, la sucesión de lecturas no puede inducir a ningún tipo de confusión: en absoluto implica que haya habido movimientos políticos de fondo, ni de aproximación, ni de debilitamiento de alguna de las posturas enfrentadas. Simplemente, la mayoría ha ido «corrigiendo» el proyecto inicial del Gobierno, matizándolo al pasar de una a otra Cámara. Para comprobarlo, baste aquí señalar que ya desde la primera lectura en la Asamblea, la aprobación contó con los votos casi unánimes de la mayoría conservadora y centrista, y el rechazo — igualmente pétreo- de la oposición de izquierdas, sin que haya apenas (como estas mismas crónicas han señalado en muchas ocasiones anteriores) «fugas» de parlamentarios aislados ${ }^{36}$. Y que esas posiciones no se han modificado, hasta el punto de que los números se repiten casi exactamente en las dos votaciones sucesivas del Senado ${ }^{37}$.

Ahora bien: como es sabido, según el artículo 89 de la Constitución gala el proceso de reforma constitucional concluye con la aprobación del texto por referéndum o, más habitualmente, por una mayoría cualificada de tres quintos de los votos emitidos por las dos Cámaras del Parlamento, reunidas en sesión conjunta del Congrès en Versalles. Así las cosas, como manifestó la votación de 2008, la progresiva «cristalización» de las posiciones en ambos polos o bloques políticos hace difícil alcanzar los tres quintos de los apoyos parlamentarios. Y, en este caso, ni la Asamblea ni el Senado llegaron a registrar un apoyo al proyecto de tres quintos de sus respectivos votantes, lo que hacía más que complicado asegurar esa misma mayoría en la votación conjunta.

36 Así, en la votación del proyecto en primera lectura, en la Asamblea Nacional, el sí se impuso por 321 votos a 299, con 6 abstenciones y un no-votante. Descendiendo al detalle, resulta que votaron a favor absolutamente todos los (20) Diputados del Nouveau Centre (NC), y 299 de los 306 de la mayoritaria Union pour un Mouvement Populaire (UMP, que registró 2 votos en contra, 4 abstenciones y el «no-voto» del Presidente de la Cámara, Bernard Accoyer). En contra, unánimes, los 25 miembros del Grupo Gauche Démocrate et Républicaine (que integra al PCF y a buena parte de los Verdes, entre otras formaciones); y 197 de los 198 del Grupo Socialiste, Radical, Citoyen et Divers Gauche (donde sólo un Diputado optó por abstenerse). Como es lógico, la división sólo se mantiene entre los Diputados no adscritos (2 favorables, 5 contrarios y una abstención, la del histórico centrista F. Bayrou). En la votación final, en tercera lectura, el Diario de Sesiones sólo recoge la aprobación global del proyecto por la Cámara (Compte rendu intégral, sesión del 13 de julio).

37 Según los respectivos Diarios de Sesiones, en primera lectura (15 de junio) el proyecto consiguió 180 votos favorables, y 151 en contra, con 8 abstenciones para totalizar 339 participantes en la votación. Pues bien: un mes después, el 11 de julio, en segunda lectura, los votos a favor y en contra fueron los mismos (180 a 151): sólo hubo un votante menos (338), que redujo las abstenciones a 7. 


\section{EL ESTADO DE LA CUESTIÓN, Y LAS INCIERTAS PERSPECTIVAS DE FUTURO}

En todo caso, el final del guión aún no está escrito. Y el debate continúa. Así, a la vuelta del verano de 2011, un destacado líder socialista (y entonces, todavía aspirante a ser el candidato de su partido a la Presidencia), Manuel Valls dirigía una «carta abierta» a los restantes precandidatos socialistas, en la que mantenía la idea de que «con su propuesta, el Presidente de la República persigue tendernos una trampa más que mejorar las finanzas públicas», pese a lo cual sugería «proponer una verdadera regla de oro, un compromiso vinculante de retorno progresivo al equilibrio» para afrontar la crisis en mejores condiciones $^{38}$. Desde el otro campo, el de la mayoría - y muy especialmente, desde la Presidencia de la República - se insistía en que las dificultades de conseguir los tres quintos del Congrès no detendrían al Presidente, cuya determinación permanecía «intacta» ${ }^{39}$.

Mientras tanto, la opinión pública comenzaba a plantearse la posibilidad de recurrir a la vía del referéndum como mecanismo para salir del impasse. Como lo expresara Alain Duhamel, la polémica sobre la «regla de oro» acabaría, si no se resuelve, por «invadir y sumergir el debate presidencial». De una parte, «la derecha pretende hacer de ella un arma electoral decisiva, en tanto la izquierda rehúsa votar afirmativamente en el Congrès para no dar al Gobierno lo que inevitablemente parecería ser una victoria e, incluso, una forma de renunciar a encarnar una vía alternativa. Sin la aprobación..., Francia estaría en una posición claramente más peligrosa. Con ella, la izquierda daría la impresión de haber sufrido una pesada derrota ideológica». Y dado que, al fin y al cabo, el Gobierno no tiene los tres quintos de los votos necesarios en el Congreso, si éste se convoca, «la regla de oro será rechazada, y la derecha lo aducirá como prueba flagrante de que la izquierda hace prevalecer sus ambiciones electorales al interés nacional». De modo que «lo único cierto es que un rechazo de la regla de oro en el Congreso desencadenaría la especulación, y una renuncia a presentar el proyecto produciría el mismo efecto».

Dado lo cual, «existe un medio de evitar esta alternativa diabólica, que es organizar un referéndum sobre la cuestión. Jurídicamente, ello no plantea ninguna dificultad»: está previsto en el artículo $89 \mathrm{CF}$ y es, pues, «perfectamente legítimo. Y políticamente, permite salir de una opción imposible o, cuanto menos, particularmente arriesgada». En este contexto, «el referéndum daría ocasión a un debate de fondo que constituiría una introducción seria a la elección presidencial... El PS podría elegir entre el sí, el no, el voto en blanco y la abstención, lo que le ofrecería una panoplia de soluciones suficientemente amplia. Se dirá que el «no» puede ganar perfectamente, como en 2005: es obvio. Pero si la regla de oro fuese rechazada, con los inconvenientes que ello implica, más valdría que los franceses lo hubiesen querido así. Y, si el «sí» se impone, Francia tendrá un arma de peso frente a los mercados y los especuladores» ${ }^{40}$.

En ese contexto de debate, las (como se ha visto, más que dudosas) perspectivas de aprobación se complicaron aún más con las elecciones senatoriales que, el 25 de sep-

38 Recogida en Libération, 22 de agosto de 2011.

39 Declaración de la portavoz del Gobierno, Valérie Pécresse (Vide www.liberation.fr, 7 de septiembre de 2011).

40 Libération, 8 de septiembre de 2011. 
tiembre de 2011, otorgaron a la izquierda (por primera vez en la V República) una mayoría en la Cámara Alta ${ }^{41}$. En ese contexto, los líderes de los partidos Socialista y de los Verdes, F. Hollande y J.-V. Placé, anunciaron esa misma noche que «la regla de oro» era la primera víctima de esas elecciones ${ }^{42}$. Todo lo cual explica que el proyecto haya quedado en un cierto punto muerto, a la espera de que las circunstancias puedan modificarse para hacer posible una aprobación definitiva que, en el momento de cerrarse estas páginas (febrero de 2012), se antoja más que complicada.

Tanto más cuanto las posiciones de la izquierda distan de modificarse. Y así, ante el Consejo Europeo del 8-9 de diciembre de 2011, en el que se adoptó la decisión de que todos los países de la Unión Europea deben constitucionalizar la «regla de oro» presupuestaria que exige un equilibrio entre ingresos y gastos públicos a lo largo del ciclo, los socialistas mantenían su negativa, denunciando que la posición francesa en esa cumbre estaba contaminada por razones de "pequeña política» nacional ${ }^{43}$. En consecuencia, posteriormente el Presidente de la República ha sido aún si cabe más incisivo en sus discursos, afirmando que Francia ha dado su palabra de incluir la regla de oro en la Constitución y que «la palabra de Francia se impone a todos» ${ }^{44}$. La posible existencia de una obligación de reformar la Constitución que se deriva de esta alocución ha sido fuertemente criticada por algún comentarista, como Pascal Jan, para quien el Jefe del Estado ha pisoteado con sus palabras «los fundamentos del Estado de Derecho, la jerarquía de poderes y de normas tal y como las concebimos desde la Revolución, desde Sieyès y más tarde Kelsen» ${ }^{45}$. En opinión de este autor, el razonamiento que esconden las palabras del Presidente es que «el legislador constitucional debe someterse a las decisiones de los dirigentes europeos porque el Presidente de la República lo ha decidido así en el marco de las negociaciones europeas. La disciplina presupuestaria definida en el plano europeo aprobada por el Jefe del Estado exigiría al poder constituyente actuar en el sentido definido por un poder constituído» ${ }^{46}$.

En definitiva, la cuestión sigue abierta, y las posturas enfrentadas, en un contexto marcado por la próxima doble convocatoria electoral, que parece otorgar escasas posibilidades de éxito al proyecto. Lo cual, obviamente, no significa que todo esté dicho. Porque, en todo caso, y salvo un cambio de circunstancias que en este momento no parece probable, la cuestión no va a desaparecer, y las mayorías surgidas de las urnas habrán de enfrentarse nuevamente a ella. A ello habremos de volver, muy probablemente, en futuras crónicas.

$$
* * *
$$

TITLE: The french debate on the constitutional reception of the golden rule of balanced budget

Resumen: La Francia de Nicolás Sarkozy aparece como uno de los principales impulsores del debate sobre la consagración constitucional de la «regla de oro» del equilibrio presupuestario, planteado en toda la Europa del euro como res-

41 Alcanzando 175 senadores sobre un total de 348.

42 Véase $w w w$.lemonde.fr, 25 septiembre 2011.

43 www.liberation.fr, 7 de diciembre de 2011.

44 Discurso de felicitación de año nuevo a los parlamentarios en el Palacio del Elíseo, 11 de Enero de 2012.

45 JAN, P, L'État constitutionnel menacé, Le Monde, 24 de enero de 2012.

46 Ibid. 
puesta a la crisis económica y a la difícil situación en los mercados de las deudas públicas nacionales. Sin embargo, y pese a que tal principio aparezca en la Constitución gala desde la última reforma de 2008, la mayoría considera necesarias algunas modificaciones constitucionales adicionales para asegurar su puesta en práctica, algo que la oposición rechaza. Ello ha suscitado una fuerte polémica, impidiendo hasta el momento su aprobación, y permite augurar que el bloqueo actual no podrá superarse, al menos, hasta la doble elección (presidencial y legislativa) que ha de celebrarse en los próximos meses.

ABSTRACT: Nicola Sarkozy's France is one of the main promoters of the public debate on the need to include in the Constitution the "golden rule» of balanced budget, posed throughout the European euro area as an instrument to fight against the economic crisis and the storm affecting the international markets with respect to the national public debts. Nonetheless, even though this principle was introduced in the French Constitution in the last 2008 reform, the present political majority considers that new constitutional changes are needed to make possible its implementation, something which the political opposition strongly denies. As a result of this opposition, these new reforms have not been approved up to now, and the debate seems likely to be blocked, at least until the next (presidential and legislative) elections, which have to be held this year.

Palabras Clave: Francia, reforma constitucional, equilibrio presupuestario, "regla de oro».

KEY WORDS: France, constitutional reform, balanced budget, «golden rule».

FECHA RECEPCIÓN: 9.02.2012 FECHA ACEPTACIÓN: 15.02.2012. 